\title{
Presence of family during CPR of Intensive Care Unit patients
}

\author{
Anis Aribogan, Sule Akin Enes, Asli Karsli \\ Baskent University Faculty of Medicine Department of Anesthesiology and Reanimation, Adana, Turkey
}

Purpose: Presence of family during CPR is allowing family to watch resuscitation. Although this concept is not familiar in Turkey, our study represents the possibility.

Materials and methods: 36 family members of ICU patients were accepted to CPR area. All CPRs were managed by director of ICU, other consultant physician prepared relatives for the situation. While CPR is continuing, consultant asked relatives if they want to be onsite and gave details about CPR. Inclusion criteria for relatives were to be parents, sisters/brothers, sons/daughters and over 24 years old. They were followed in terms of reactions, emotional status, welcoming results, satisfactions and educational status were noted as well.

Results: Causes were trauma for 6 (16.7\%), cerebral aneurysm for $4(11.1 \%)$, intraabdominal sepsis for $8(22.2 \%)$, suicidal intoxication for 1 (2.8\%), thoracic surgery for 3 (8.3\%), terminal cancer disease for 14 (38.9\%) patients. 2 patients were under 10, 4 between 10-20, 16 between 20-40, 12 between 40-60 and 2 more than 60 years old (Table I). $14(38.9 \%)$ of relatives were parents, 15 (41.7\%) sisters/brothers, 7 (19.4\%) sons/daughters. $25(69.4 \%)$ have been graduated from university whereas $9(25 \%)$ from high, 2 (5.6\%) from elementary schools. Only three members showed exaggerated reactions and obstructed the administration (Table II). They were female parents from rural area, 1 of them have been graduated from elementary and others from high schools. All relatives remained on site were extremely satisfied.
Conclusions: Family members who wants to standby with their beloved ones when they are so closed to death, the selected ones should be permitted. Excessive reactions may be due to gender, education, residence. On the conditions of well done and self confident CPR, it will be easy for everyone to handle results.

Table I. Demographics of Cardiac Arrest Patients

\begin{tabular}{|l|l|l|l|}
\hline Ages & \multicolumn{2}{|l|}{ Genders } & Causes of Cardiac Arrest \\
\hline $\begin{array}{l}<10 \text { years } \\
(n=2)\end{array}$ & $\begin{array}{l}\text { Female } \\
(n=1)\end{array}$ & $\begin{array}{l}\text { Male } \\
(n=1)\end{array}$ & $\begin{array}{l}\text { Trauma } \\
(n=6,16.7 \%)\end{array}$ \\
\hline $\begin{array}{l}10-20 \text { years } \\
(n=4)\end{array}$ & $\begin{array}{l}\text { Female } \\
(n=3)\end{array}$ & $\begin{array}{l}\text { Male } \\
(n=1)\end{array}$ & $\begin{array}{l}\text { Cerebral aneurysm } \\
(n=4,11.1 \%)\end{array}$ \\
\hline $\begin{array}{l}20-40 \text { years } \\
(n=16)\end{array}$ & $\begin{array}{l}\text { Female } \\
(n=7)\end{array}$ & $\begin{array}{l}\text { Male } \\
(n=9)\end{array}$ & $\begin{array}{l}\text { Intraabdominal sepsis } \\
(n=8,22.2 \%)\end{array}$ \\
\hline $\begin{array}{l}40-60 \text { years } \\
(n=12)\end{array}$ & $\begin{array}{l}\text { Female } \\
(n=8)\end{array}$ & $\begin{array}{l}\text { Male } \\
(n=4)\end{array}$ & $\begin{array}{l}\text { Suicidal intoxication } \\
(n=1,2.8 \%)\end{array}$ \\
\hline$>60$ years & Female & Male & Thoracic surgery \\
$(n=2)$ & $(n=0)$ & $(n=2)$ & $(n=3,8.3 \%)$ \\
\hline & & $\begin{array}{l}\text { Terminal Ca disease } \\
(n=14,38.9 \%)\end{array}$ \\
\hline
\end{tabular}

Table II. Characteristics of the Families

\begin{tabular}{|l|l|l|}
\hline Relations & Educational Status & Reactions \\
\hline Parents & University & Exaggerated \\
$(n=14,38.9 \%)$ & $(n=25,69.4 \%)$ & $(n=3,8.3 \%))$ \\
\hline $\begin{array}{l}\text { Sisters/Brothers } \\
(n=15,41.7 \%)\end{array}$ & High School & Crying \\
$(n=9,25 \%)$ & $(n=26,72.2 \%)$ \\
\hline $\begin{array}{l}\text { Sons/Daughters } \\
(n=7,19.4 \%)\end{array}$ & $\begin{array}{l}\text { Elementary School } \\
(n=2,5.6 \%)\end{array}$ & $\begin{array}{l}\text { Calm } \\
(n=7,19.5)\end{array}$ \\
\hline
\end{tabular}

